\title{
The Distance and Distribution of Galactic Supernova Remnants from the PMN Survey Sample
}

\author{
M. Stupar, ${ }^{1}$ M. D. Filipović, ${ }^{2,3}$ T. G. Pannuti ${ }^{4}$ and Q. A. Parker ${ }^{1,5}$ \\ ${ }^{1}$ Department of Physics, Macquarie University, Sydney, NSW 2109, \\ Australia \\ ${ }^{2}$ University of Western Sydney, Locked Bag 1797, Penrith South DC, \\ NSW 1797, Australia \\ ${ }^{3}$ Australia Telescope National Facility, CSIRO, P.O. Box 76, Epping, \\ NSW 1710, Australia \\ ${ }^{4}$ MIT Center for Space Research, 77 Massachusetts Avenue NE80-6015, \\ Cambridge, MA 02139, USA \\ ${ }^{5}$ Anglo-Australian Observatory, P.O. Box 296, Epping, NSW 1710, \\ Australia
}

\begin{abstract}
We have used the Parkes-MIT-NRAO (PMN) radio survey, at a common frequency of $4.85 \mathrm{GHz}(\lambda=6 \mathrm{~cm})$, to study the distribution of Galactic shell supernova remnants (SNRs). We estimate distances and distribution of the Galactic shell SNRs and find the peak of distances to be between 4 and $6 \mathrm{kpc}$. Our two-dimensional model of the distribution of Galactic shell SNRs shows a concentration between the Sagittarius and the Scutum arms.
\end{abstract}

\section{Introduction}

The PMN Survey, at $4.85 \mathrm{GHz}$, was undertaken with three radio telescopes: Green Bank's 91-m and 43-m telescopes were used to scan the northern sky (Condon et al. 1989, 1991), and the Parkes 64-m telescope (Condon et al. 1993), was used to scan the southern sky (at the same frequency).

The angular sizes of supernova remnants (SNRs) are taken from Green (2001) and used in the estimation of flux densities for SNRs recognized in the PMN. The surface brightness (calculated for $4.85 \mathrm{GHz}$ ) and diameter (in pc) for the shell SNRs was used to establish the $\Sigma-D$ relation (Shklovsky 1960). The derived distances and distribution of the shell SNRs was then considered.

\section{Observations and Data Analysis}

The data have been downloaded from the Australia Telescope National Facility (ATNF) and SkyView Virtual Observatory WWW pages. ${ }^{1}$ Since the PMN survey is performed with single dish telescopes, while SNRs are mostly extended

${ }^{1}$ See ftp://ftp.atnf.csiro.au/pub/data/pmn/surveys and http://skyview.gsfc.nasa.gov. 
objects, we had to take special care with SNRs extended in the north-south direction. This is because applying correction in spillover temperature variation reduces the fluxes (Condon et al. 1991, 1993). The 43-m telescope observations are used only for fields not covered by Parkes.

\section{Distance to Galactic Shell SNRs}

For Galactic shell SNRs, with only radio observations, it is common to use the $\Sigma-D$ relation, find the power law index, $\beta$, and then estimate distances. Our calculations showed that shells concentrate between 2 and $8 \mathrm{kpc}$ with a peak between 4 and $6 \mathrm{kpc}$. Leahy \& Xinji (1989) also found a peak value between 4 and $6 \mathrm{kpc}$ and Case \& Bhattacharya (1998) estimated the peak distribution at $\sim 5 \mathrm{kpc}$ with a scale length of $\sim 7 \mathrm{kpc}$.

Also, we have examined the height, $|z|$, of shell SNRs relative to the Galactic plane. It is clear that the highest concentration of shells is $\sim \pm 200 \mathrm{pc}$ from the plane, and that the remnants G93.3+6.9 and G55.7+3.4 have the highest values of $|z|, 673 \mathrm{pc}$ and $604 \mathrm{pc}$, respectively.

\section{Galactic Distribution of the Shell SNRs}

Using a Galactocentric distance of $8.5 \mathrm{kpc}$, and spiral arms as defined by Russeil (2003), we found a concentration of SNRs towards the Galactic Center (between Galactic longitudes -40 to 40 degrees), as expected. This also showed that shells in the Galaxy are largely distributed between the Sagittarius and Centaurus arms. Similarity was found between the distribution of Galactic SNRs, and that of $\mathrm{H}$ II regions as presented by Paladini et al. (2004).

Acknowledgments. We are thankful to the ATNF for use of PMN South FITS files and of the Karma and MIRIAD data analysis software packages, and NASA's SkyView for providing PMN North FITS files.

\section{References}

Case, G. L., \& Bhattacharya D. 1998, ApJ, 504, 761

Condon, J. J., Broderick, J. J., \& Seielstad, G. A. 1989, AJ, 97, 1064

- 1991, AJ, 102, 2041

Condon, J. J., Griffith, M. R., \& Wright, A. L. 1993, AJ, 106, 1095

Green, D. A. 2001, A Catalogue of Galactic SNRs, (Cambridge: MRAO)

Leahy, D. A., \& Xinij, W. 1989, PASP, 101, 607

Paladini, R., Davies, R. D., \& DeZotti, G. 2004, MNRAS, 347, 237

Russeil, D. 2003, A\&A, 397, 133

Shklovsky, I. S. 1960, Sov. Astr., 4, 243 